\title{
McXtrace 1.4: latest developments in the new release
}

Knudsen, Erik B.; Willendrup, Peter Kjær; Garde, Jakob; Jørgensen, Mads R. V.; Kantor, Innokenty

Published in:

Proc. SPIE 10403, Infrared Remote Sensing and Instrumentation XXV

Link to article, DOI:

$10.1117 / 12.2274416$

Publication date:

2017

Document Version

Publisher's PDF, also known as Version of record

Link back to DTU Orbit

Citation (APA):

Knudsen, E. B., Willendrup, P. K., Garde, J., Jørgensen, M. R. V., \& Kantor, I. (2017). McXtrace 1.4: latest developments in the new release. In Proc. SPIE 10403, Infrared Remote Sensing and Instrumentation XXV (Vol. 10388). SPIE - International Society for Optical Engineering. Proceedings of SPIE - The International Society for Optical Engineering https://doi.org/10.1117/12.2274416

\section{General rights}

Copyright and moral rights for the publications made accessible in the public portal are retained by the authors and/or other copyright owners and it is a condition of accessing publications that users recognise and abide by the legal requirements associated with these rights.

- Users may download and print one copy of any publication from the public portal for the purpose of private study or research.

- You may not further distribute the material or use it for any profit-making activity or commercial gain

- You may freely distribute the URL identifying the publication in the public portal 


\section{McXtrace 1.4: latest developments in the new release}

Erik B. Knudsen, Peter K. Willendrup, Jakob Garde, Mads R. V. Jørgensen, Innokenty Kantor

Erik B. Knudsen, Peter K. Willendrup, Jakob Garde, Mads R. V. Jørgensen, Innokenty Kantor, "McXtrace 1.4: latest developments in the new release," Proc. SPIE 10388, Advances in Computational Methods for X-Ray Optics IV, 1038809 (23 August 2017); doi: 10.1117/12.2274416

SPIE Event: SPIE Optical Engineering + Applications, 2017, San Diego, California, United States 


\title{
McXtrace 1.4: latest developments in the new release.
}

\author{
Erik B. Knudsen ${ }^{a}$, Peter K. Willendrup ${ }^{a}$, Jakob Garde ${ }^{a}$, Mads R. V. Jørgensen ${ }^{b, c}$, Innokenty \\ Kantor $^{a, c}$ \\ ${ }^{a}$ Physics Dept., Technical University of Denmark, Kgs. Lyngby, Denmark; \\ ${ }^{b}$ Dept. of Chemistry \& iNANO-center, Aarhus University, Aarhus, Denmark; \\ ${ }^{c}$ MAX-IV Laboratory, Lund, Sweden
}

\begin{abstract}
This paper presents details on some of the important new features in the newly released version of the x-ray tracing software package McXtrace. Although many developments have been made, this presentation is focused on the features that were required to meet the challenges posed for accurate simulation of the DanMAX beamline - a beamline currently under design at the MAX IV synchrotron. Among these may be mentioned: new sourcemodels, new monochromator crystal models, multilayer capabilities, and the full beamline simulation frame itself.
\end{abstract}

Keywords: X-ray, simulation, ray tracing, software

\section{INTRODUCTION}

McXtrace $^{1,2}$ is an open source, $\mathrm{x}$-ray tracing software package designed for simulating any and every $\mathrm{x}$-ray experiment and instrumentation. It was initially released in 2009, but builds on the solid foundation of McStas, ${ }^{3}$ a very similar tool for neutron ray tracing with a significantly longer history and a proven record. In fact the tools share much of the same code base and workflow. Thus, any user familiar with either can easily switch to the other.

McXtrace operates within the realm of geometrical optics, where ray tracing is directly valid, but since it also tracks the phase of the photons as they travel through the simulation it may be coerced into also handling effects like slit diffraction, etc. ${ }^{1}$

A new version, 1.4, of the package has recently been released, which includes numerous improvements over the old version. In summary, the new release includes:

1. A new set of modern, python based GUI and plotting tools

2. Many new components. See section 2 below.

3. An interface to the MCPL-file format. ${ }^{4}$

4. A homogenization of components of similar type, to make it easier for users to switch between models.

5. More standardized installation on debian ${ }^{5}$ class systems.

6. New example simulations.

7. Support for the NeXus-file format

The following is a review of some of the main things that are included in the present release. Firstly, we present a couple of new realistic source models, secondly some optical components, and lastly we show a complete example of beamline simulation: the materials science beamline DanMAX at the MAX IV Synchrotron in Lund, Sweden. The review is focused on the new features that have been built in the process of setting up this complete model of DanMAX.

Further author information: (Send correspondence to E.B.K.)

E.B.K.: E-mail: erkn@fysik.dtu.dk, Telephone: +45 21326655

Advances in Computational Methods for X-Ray Optics IV, edited by Oleg Chubar,

Kawal Sawhney, Proc. of SPIE Vol. 10388, 1038809 · C 2017 SPIE

CCC code: $0277-786 X / 17 / \$ 18 \cdot$ doi: $10.1117 / 12.2274416$

Proc. of SPIE Vol. 10388 1038809-1 


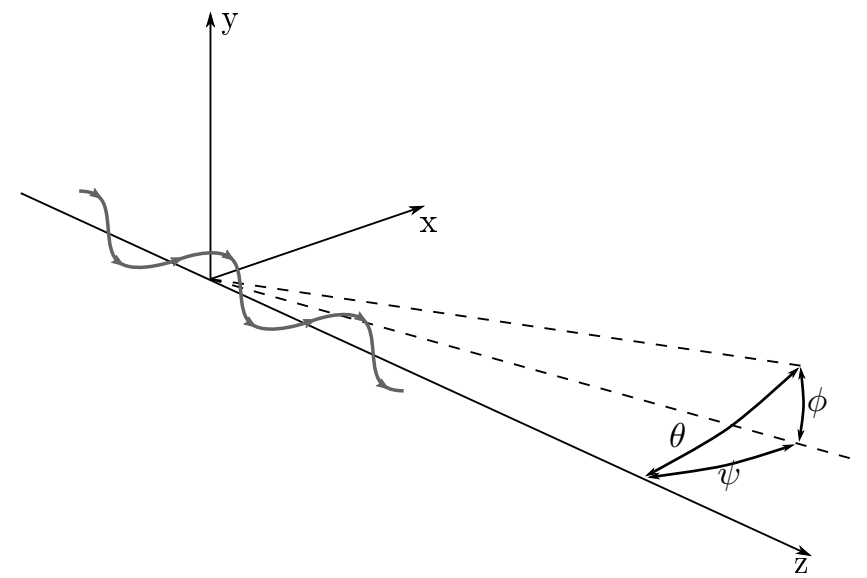

Figure 1. Sketch defining of the parameters $\theta, \phi$, and $\psi$ from eq. (1)

\section{NEW SOURCE COMPONENT MODELS}

This section will describe a subset of the new x-ray source component models present in McXtrace version 1.4. In general we find that the new McXtrace models of undulator and bending magnet agrees well with computations performed using other software packages.

\subsection{Undulator}

The recently included undulator source model is based on the model developed by Kim. ${ }^{6}$ The master equation governing the simulated undulator radiation (in terms of differential flux) is taken to be:

$$
\frac{\mathrm{d}^{2} F_{\sigma, \pi}}{\mathrm{d} \Omega \mathrm{d} \omega}=\alpha \frac{I}{e}\left(\frac{K \gamma}{1+K^{2} / 2}\right)^{2}\left(\frac{\omega}{\omega_{1}(0)}\right)^{2} N^{2} S_{N}\left(\omega / \omega_{1}(\theta)\right) B_{\sigma, \pi}^{2}(\omega, \phi, \psi)
$$

where $K$ is the dimensionless undulator parameter, $\alpha$ is the fine structure constant, $I$ the storage ring current, $e$ the ring energy, and $\gamma$ the Lorentz factor. Figure 1 shows the definition of the coordinate system for the radiation and the angle parameters $\theta, \phi$, and $\psi$. Furthermore: $\omega_{1}(\theta)=\frac{2 \gamma^{2}}{1+K^{2} / 2+\gamma^{2} \theta^{2}} \omega_{u}$ is a corrected fundamental angular frequency of the undulator.

The last terms in eq. (1) are defined by eqs. (2) to (4):

$$
S_{N}\left(\omega / \omega_{1}(\theta)\right)=\left(\frac{\sin N \pi \omega / \omega_{1}(\theta)}{N \sin \pi \omega / \omega_{1}(\theta)}\right)^{2}
$$

and

$$
\left(\begin{array}{c}
B_{\sigma}(\omega, \phi \psi) \\
B_{\pi}(\omega, \phi \psi)
\end{array}\right)=\frac{1}{\pi} \int_{-\pi}^{\pi}\left(\begin{array}{c}
\psi / K-\cos \xi \\
\phi / K
\end{array}\right) \exp i\left(\left(\omega / \omega_{1}(\theta)\right) \xi-p \sin \xi+q \sin 2 \xi\right)
$$

with

$$
p(\xi)=2 \frac{\omega}{\omega_{1}(0)} \frac{\phi \gamma K}{1+K^{2} / 2}, \quad q(\xi)=\frac{1}{4} \frac{\omega}{\omega_{1}(0)} \frac{K^{2}}{1+K^{2} / 2}
$$

To evaluate the integral in eq. (3), we have used the quadrature routines available from the GNU Scientific Library. ${ }^{7}$ To do so we utilize a new feature of McXtrace, where component developers may easily connect to external libraries through the DEPENDENCY-keyword, in the component definition file. In the undulator definition this is is written as:

DEPENDENCY "-lgs $l_{\smile}-\operatorname{lgs} \mathrm{c}_{\mathrm{c}} \mathrm{b}$ as $"$ 


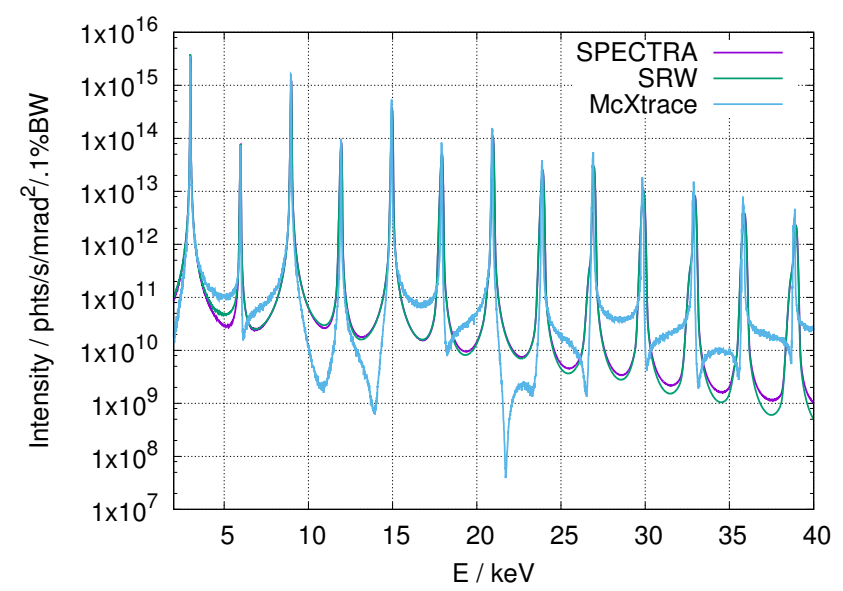

Figure 2. Simulated undulator spectrum for the proposed DanMAX-undulator tuned to a peak at $15 \mathrm{keV}$. Purple) Sim. with SPECTRA; ${ }^{11}$ green) Sim. w. SRW; ${ }^{9}$ blue) Sim. w. McXtrace. ${ }^{2}$

In the creation process the model was tested against proven $\operatorname{codes}^{8,910,11}$ which are used extensively for undulator radiation. Figure 2 shows a comparison of the radiation spectrum detected $20 \mathrm{~m}$ downstream from an undulator with the same parameters as the one currently under order for the DanMAX ${ }^{12}$ (IVU16) (See section 4) beamline at MAX IV Laboratory. ${ }^{13}$ This undulator is $\approx 3 \mathrm{~m}$ long, with a period of $16 \mathrm{~mm}$, corresponding to 187 undulator periods. For this test, the undulator was tuned to emit radiation at $15 \mathrm{keV}$. Given the ring parameters of the $3 \mathrm{GeV}$ storage ring at MAX IV, this sets the undulator gap to $\approx 6.01 \mathrm{~mm}$, which in turn yields a peak magnetic field at the undulator centre of $0.8363 \mathrm{~T}$ (or in terms of undulator parameter $K=1.249$ ). On-peak characteristics match quite well across computer codes, whereas the description at half periods differs significantly. In many cases this accuracy is quite sufficient owing to the high ratio between peak and valley flux.

To include emittance effects originating from an imperfect electron beam in the storage ring we employ a simple Monte Carlo scheme in line with the general philosophy and design of McXtrace (and other x-ray tracing tools), and simply add normally distributed errors to both the origin of the ray and its direction. In terms of fig. 1, this amounts to adding normally distributed errors to the origin and the angles $\phi$ and $\psi$. We have validated the procedure against computations with identical parameters in SPECTRA and found the result to agree satisfactorily (See figs. 3 and 4 ).

\subsection{Bending Magnet}

Further to the completeness of the set of available source models in McXtrace, we distribute a bending magnet source. Based on a simplified approach ${ }^{14}$ it is very efficient and provides a fairly accurate description of radiation from a bending magnet source within the central cone of radiation. A comparison between the McXtrace description and simulations using SPECTRA is shown in fig. 5. Clearly, the two descriptions agree to a very high degree.

\section{NEW OPTICS COMPONENTS}

In this section we present some of the the main new/improved optics components in McXtrace.

\subsection{Mirrors}

The distribution now includes models of mirrors including an easy to use flat perfectly flat mirror, as well as a toroidally bent mirror. The toroid my be focusing or defocusing depending on the signs of the bending radii. This is useful e.g. when the effect of heat-load induced bumps is investigated. All mirrors can now take as input an external reflectivity file, which may be resolved in energy and incidence angle or simply q. This gives the simulator the option of computing reflectivities using specialized software, e.g. IMD which has found widespread use in the astrophysics community. Through this we can easily include multilayer coatings in the simulations. 

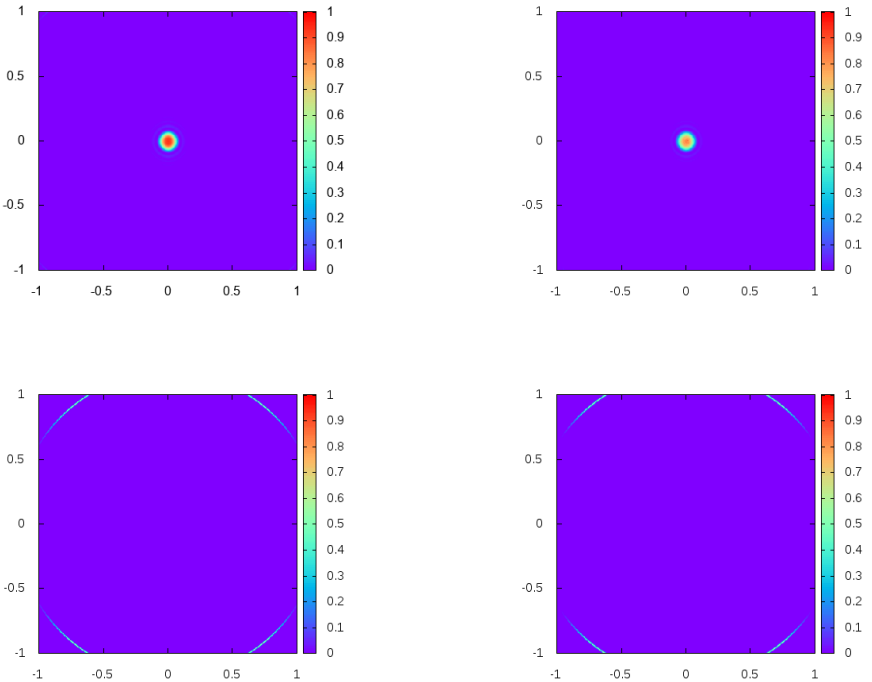

Figure 3. Comparison of the spatial distribution of undulator radiation $20 \mathrm{~m}$ downstream from an undulator of the IVU16 type in the MAX-IV storage ring, where the undulator has been tuned to have its 11 th harmonic peak at $33 \mathrm{keV}$. The left column is calculations done in SPECTRA, the right is done with the undulator model in McXtrace. Top row is on-peak radiation $(33 \mathrm{keV})$, bottom row is an example of off-peak radiation at $25.3 \mathrm{keV}$. The peak intensity has been normalized to 1 .

\section{$X, Y / \mathrm{mm}$}
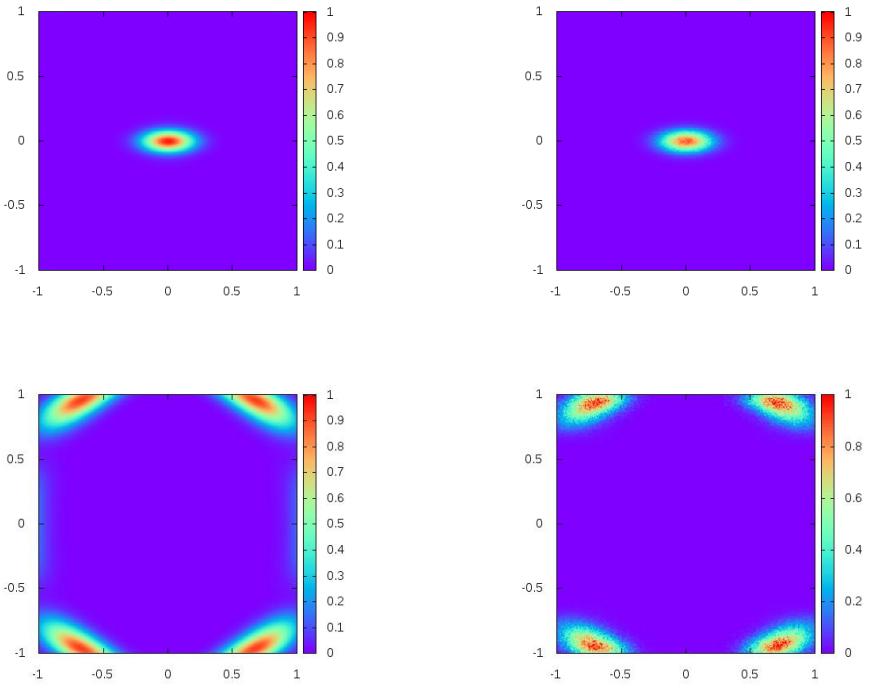

Figure 4. Comparison of the spatial distribution of undulator radiation, with the inclusion of emittance from an imperfect electron beam, measured $20 \mathrm{~m}$ downstream from an undulator of the IVU16 type in the MAX-IV storage ring, where the undulator has been tuned to have its 11 th harmonic peak at $33 \mathrm{keV}$. The left column is calculations done in SPECTRA, the right is done with the undulator model in McXtrace. Top row is on-peak radiation ( $33 \mathrm{keV})$, bottom row is an example of off-peak radiation at $25.3 \mathrm{keV}$. The peak intensity has been normalized to 1 . 


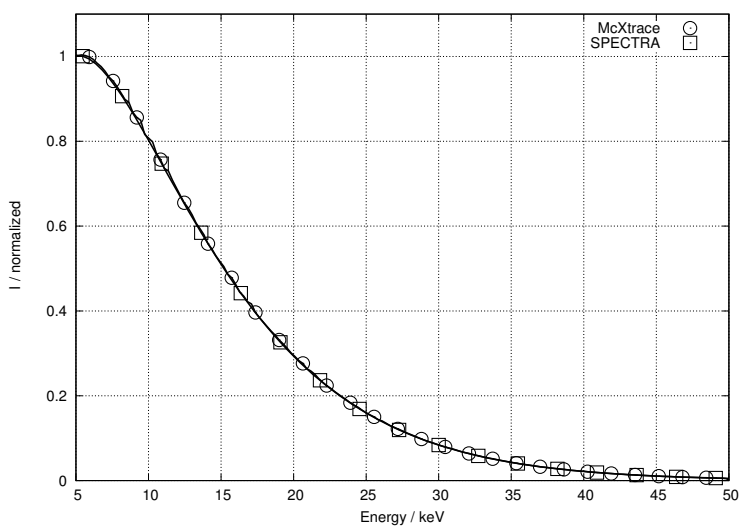

Figure 5. Comparison between energy spectra of SPECTRA (squares) and McXtrace (circles) bending magnet models. The bending magnet is assumed to have a $0.6 \mathrm{~T}$ and to be in a $2.4 \mathrm{GeV}, 0.4 \mathrm{~mA}$ storage ring. Energy spread and emittance effects have been neglected.

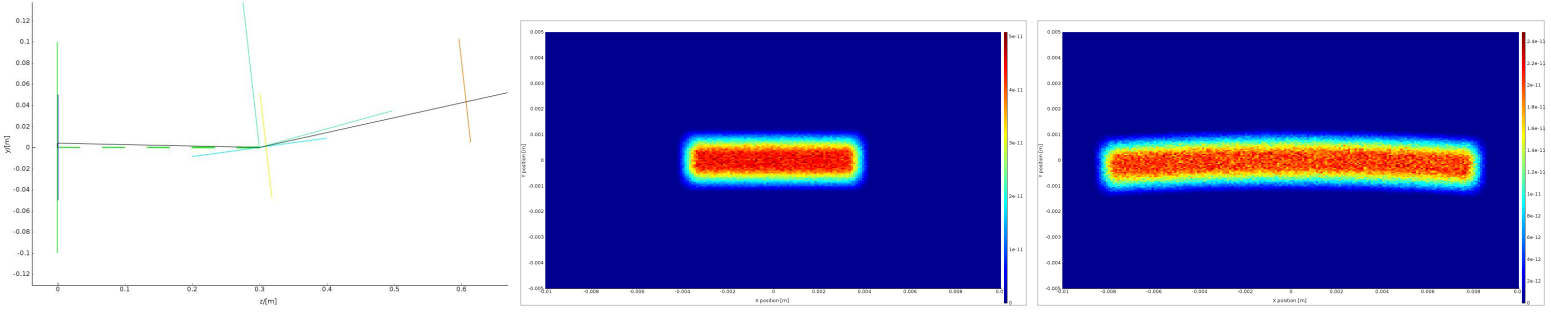

Figure 6. Left) Sketch of a raytracing example with mirror directly from the mxdisplay-tool shipped with McXtrace. The area plots (middle and right) show the intensity distribution of a flat uniform source reflected in a mirror and monitored $1 \mathrm{~m}$ downstream. The edges are not sharp in either plot due to divergence in the source model. Middle) Flat mirror. Right) Toroidal mirror with radii $20 \mathrm{~m}$ and $0.01 \mathrm{~m}$.

The model of the DanMAX-beamline (See section 4.) includes a double bounce multilayer monochromator, where the first element is modelled by interchangeable bent and flat mirrors. The former is used where the full white beam impinges on the first multilayer resulting in a heat-bump; the latter when the beam has been pre-monochromatized and no heat-bump is generated. A bottleneck when simulating mirrors with coatings, has been file IO, in particular for multilayers where high energy and angle resolution is often required. This is now less cumbersome due to a scheme where a datafile may be shared among otherwise independent beamline components.

\subsection{Monochromator Crystals}

Simulating monochromator crystals in McXtrace may be done using the two new components Bragg_crystal.comp and Bragg_crystal_bent.comp. The models are currently limited to single-atom crystals with cubic spacegroups. We have compared the characteristics of crystals models against $\mathrm{XOP}^{15}$ and analytical calculations ${ }^{16}$ which generally shows very good agreement as shown in fig. 7. A small offset to the peak location, on the order of $0.1 \mathrm{eV}$, remains between the perfectly flat crystal models. This may be attributed to different crystallographic standards in XOP and McXtrace, when describing the atomic distances of crystalline Si.

\section{SIMULATION EXAMPLE: DANMAX}

As a tool in the design process of the DanMAX beamline at the MAX IV synchrotron, we have developed a complete McXtrace-model of the beamline. The model has helped make decisions on various issues, and is tracking design changes as they happen (for instance due to engineering realities). Figure 8 shows a sketch of the beamline layout, where the central components have been included. The beamline will serve as both an imaging as well as a powder diffraction beamline. A unique feature of this beamline is the use of a double bounce multilayer monochromator (DMM) in conjunction with a standard high-resolution double crystal monochromator 


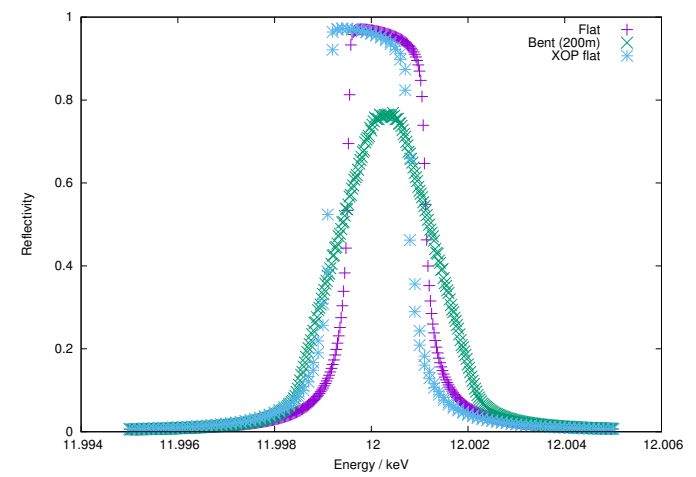

Figure 7. Spectra of radiation reflected by a $\mathrm{Si} 111$-monochromator tuned to $12 \mathrm{keV}$. The radiation illuminating the crystals is uniformly distributed in energy and fully collimated. Purple corresponds to a perfectly flat crystal, green to a crystal bent to a radius of $200 \mathrm{~m}$, and blue to a flat crystal simulated by XOP. The slight difference in central energy is due to mismatching crystallographic descriptions of Si.

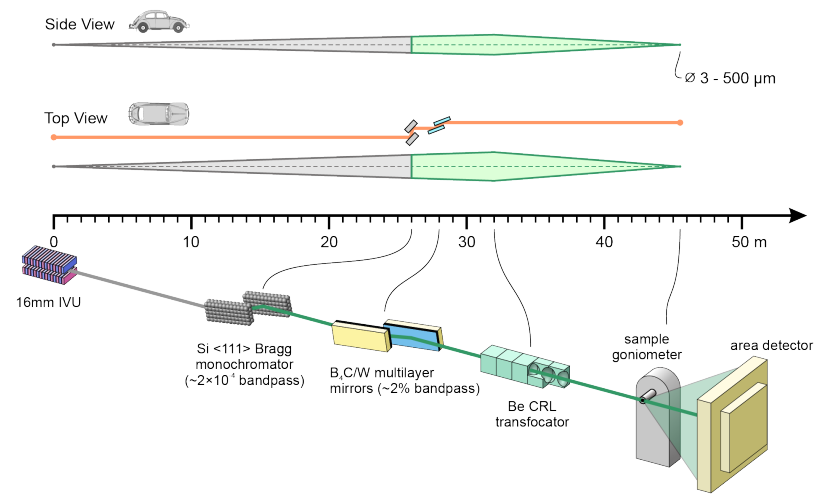

Figure 8. Cartoon of the DanMAX beamline optical concept.

(DCM). When the DCM is active the DMM may be used for higher harmonic suppression. To exemplify: if the DCM is tuned to $15 \mathrm{keV}$ simulations indicate that the harmonic at $45 \mathrm{keV}$ is suppressed by 11 orders of magnitude (fig. 9). Figure 10 shows examples of data that may be expected at DanMAX where the real beamline parameters for e.g. source, detector pixels, heat load corrections etc. have been included.

\section{INFRASTRUCTURE IMPROVEMENTS}

\subsection{New GUI}

A new Python/Qt based GUI is available for McXtrace. Section 5.1 shows examples of the new simulation GUI (left) and of the new plotting tool. Also included in the new GUI is an improved editor based on QScintilla. A novel feature is the predictive text editors helping users write instrument files.

\section{ACKNOWLEDGEMENTS}

This project was partly funded by the DanMAX project (Danish Ministry for Higher Education and Science, the Capital Region, the Central Denmark Region, MAX IV Laboratory, Technical University of Denmark, Aarhus University and University of Copenhagen).

\section{REFERENCES}

[1] Bergbäck Knudsen, E., Prodi, A., Baltser, J., Thomsen, M., Kjær Willendrup, P., Sanchez del Rio, M., Ferrero, C., Farhi, E., Haldrup, K., Vickery, A., Feidenhans'l, R., Mortensen, K., Meedom Nielsen, M., 


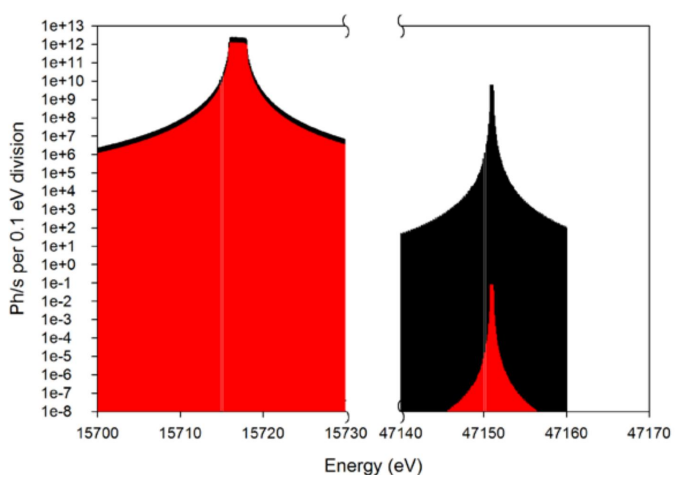

Figure 9. Higher harmonic suppression using a double bounce multilayer monochromator. Double crystal monochromator is tuned to a Bragg angle corresponding to $15 \mathrm{keV}$. The black curve shows the simulated photon flux without the double bounce multilayer monochromator, the red curves show the flux with the multilayer in. We find a higher harmonic rejection ratio of $\approx 10^{14}$.
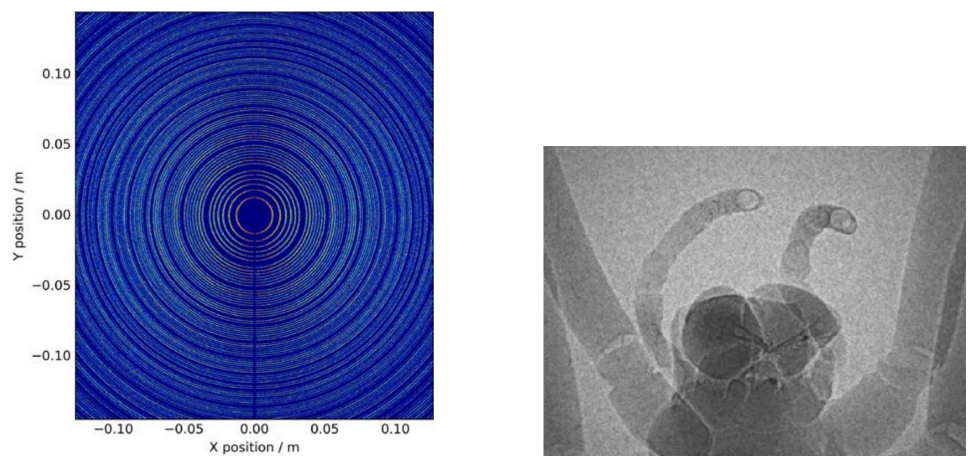

Figure 10. Examples of results obtained with the virtual DanMAX-beamline as simulated with McXtrace. Left) Standard powder diffraction pattern from an $\mathrm{LaB}_{6}$-powder. Right) Absorption contrast image of the head of a spider.
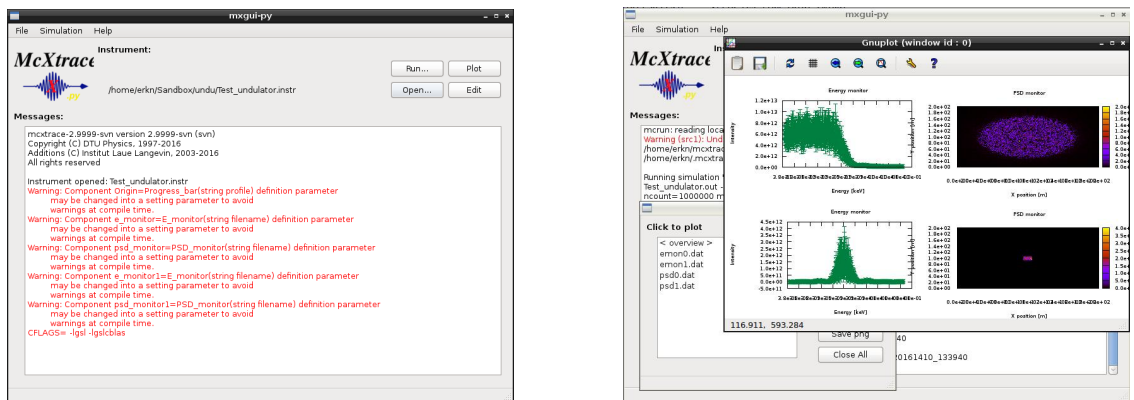

Figure 11. Examples of the new McXtrace python/Qt based GUI. Left) The main window. Right) plotting windows. 
Friis Poulsen, H., Schmidt, S., and Lefmann, K., "McXtrace: a Monte Carlo software package for simulating X-ray optics, beamlines and experiments," Journal of Applied Crystallography 46, 679-696 (Jun 2013).

[2] "Mcxtrace website," (2017).

[3] "Mcstas website," (2017).

[4] Kittelmann, T., Klinkby, E., Knudsen, E. B., Willendrup, P., Cai, X. X., and Kanaki, K., "Monte carlo particle lists: Mcpl," Computer Physics Communications 218, 17-42 (2017).

[5] "Debian website."

[6] Kim, K.-J., "Characteristics of synchrotron radiation," AIP Conference Proceedings 184, 565-632 (1989).

[7] Gough, B., [GNU Scientific Library Reference Manual - Third Edition], Network Theory Ltd., 3rd ed. (2009).

[8] Chubar, O. and Elleaume, P., "Accurate and efficient computation of synchrotron radiation in the near field region," in [proc. of the EPAC98 Conference], 1177-1179 (1998).

[9] "Srw github site."

[10] Tanaka, T. and Kitamura, H., "SPECTRA: a synchrotron radiation calculation code," Journal of Synchrotron Radiation 8, 1221-1228 (Nov 2001).

[11] "Spectra," (2016).

[12] "Danmax website."

[13] "Max iv laboratory."

[14] Patterson, B. D., "A simplified approach to synchrotron radiation," American Journal of Physics 79, 1046 (2011).

[15] del Río, M. S. and Dejus, R. J., "Xop v2. 4: recent developments of the x-ray optics software toolkit," in [Proc. SPIE], 8141, 814115 (2011).

[16] Als-Nielsen, J. and McMorrow, D., [Elements of modern X-ray physics], Wiley (2011). 